\title{
CALR Mutation Underlying Silent Stroke
}

\author{
Rehman Faryal $^{1}$ Lisa Lee Tokar ${ }^{2}$ Stephen E. Langabeer ${ }^{2}$ Janusz Krawczyk ${ }^{1}$
}

${ }^{1}$ Department of Haematology, Galway University Hospital, Galway, Ireland

${ }^{2}$ Cancer Molecular Diagnostics, St. James's Hospital, Dublin, Ireland

TH Open 2021;5:e174-e175.

Essential thrombocythemia (ET) is one of the classical Philadelphia chromosome-negative myeloproliferative neoplasms (MPN) characterized by a high platelet count and a tendency for thrombotic and/or hemorrhagic events. ${ }^{1}$ ET is driven by acquired mutations in either the JAK2, CALR, or MPL genes with the JAK2 V617F mutation a significant risk factor for development of stroke in patients with MPN. ${ }^{2}$ In contrast, CALR-mutated ET is associated with higher platelet counts but a lower risk of thrombotic events than their JAK2 V617Fpositive counterparts. ${ }^{3}$ Furthermore, patients with type 1 CALR mutations (52 base-pair deletion) appear to have an increased risk of thrombosis compared with patients harboring type 2 CALR mutations (five base-pair insertion). ${ }^{4,5}$ This aspect has been further illustrated in the molecular screening of large cohorts of patients with stroke (with and without an hematologically overt MPN) that have shown no evidence of the common ET-associated CALR mutation types. ${ }^{6,7}$
Address for correspondence Stephen E. Langabeer, PhD, FRCPath, Cancer Molecular Diagnostics, Trinity Translational Medicine Institute, St. James's Hospital, Dublin, D08 W9RT, Ireland (e-mail: slangabeer@stjames.ie).

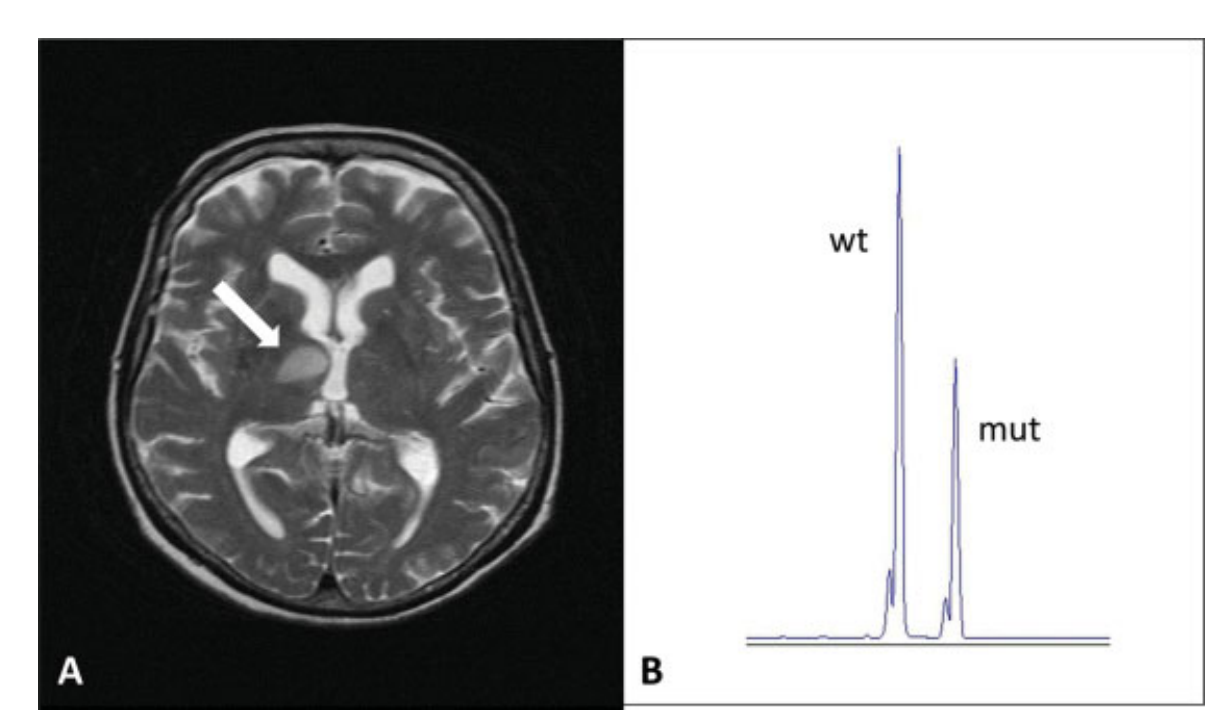

A 78-year-old female presented in the emergency department with a new onset parieto-occipital headache and recurring episodes of dizziness. She was recently diagnosed with hypertension, which was well controlled with an antihypertensive. On examination, she was vitally stable with no neurological findings. Computed tomography (CT) scan of the brain showed an area of hypoattenuation in the right thalamus that was confirmed as an acute infarct by magnetic resonance imaging (MRI) (-Fig. 1A). Cardiac workup including carotid Doppler that revealed patent carotid arteries and echocardiography that demonstrated good systolic function, no valvular defects and normal atrial dimensions. The patient remained on telemetry for rhythm monitoring as part of the stroke workup that suggested normal sinus rhythm. In view of the CT and MRI findings and lack of any obvious neurological deficit, the picture was consistent with a "silent" stroke. Hematological workup showed hemoglobin of $13.3 \mathrm{~g} / \mathrm{dL}$, white cell count of $7.5 \times 10^{9} / \mathrm{L}$

Fig. 1 (A) Brain magnetic resonance imaging depicting acute infarct (arrow). (B) Capillary electrophoresis after fragment length analysis showing a wild-type 263 base-pair peak and mutant 268 base-pair peak in CALR exon 9.

received

October 23, 2020

accepted after revision

January 26, 2021
DOI https://doi.org/

$10.1055 / \mathrm{s}-0041-1728674$.

ISSN 2512-9465.

\section{(C) 2021. The Author(s).}

This is an open access article published by Thieme under the terms of the Creative Commons Attribution License, permitting unrestricted use, distribution, and reproduction so long as the original work is properly cited. (https://creativecommons.org/licenses/by/4.0/)

Georg Thieme Verlag KG, Rüdigerstraße 14, 70469 Stuttgart, Germany 
and platelet count of $1,163 \times 10^{9} / \mathrm{L}$ (normal range $=150$ $400 \times 10^{9} / \mathrm{L}$ ). The blood film showed platelet anisocytosis, but no evidence of dacrocytes. A thrombophilia screen was not indicated according to local and national guidelines given the patients' age, no history of thrombosis and no family history of thrombosis. The patient refused a bone marrow biopsy with the diagnosis more consistent with ET rather than pre-fibrotic myelofibrosis due to the absence of anemia, leukocytosis, a leucoerythroblastic blood film, palpable splenomegaly, and a raised lactate dehydrogenase (200 IU/L; normal range 135-214 $\mathrm{IU} / \mathrm{L})^{8}{ }^{8}$ The JAK2 V617F mutation was not detected, but fragment length analysis followed by capillary electrophoresis revealed the presence of the common five base-pair insertion (type 2) mutation in CALR exon 9 at an allele frequency of $36.2 \%$, providing further evidence of ET ( - Fig. 1B). After consultation with stroke team, the patient was started on aspirin and given the degree of thrombocytosis; cytoreduction therapy with hydroxyurea was initiated. The patients' symptoms and platelet count improved over the next few days with hydroxyurea continued.

Despite previous studies revealing no evidence, this case demonstrates that CALR mutations can exist in patients presenting with stroke and a reasonable suspicion of ET; a situation similar to the very low incidence of CALR mutations in patients presenting with splanchnic vein thrombosis. ${ }^{9}$ CALR mutational analysis should therefore be considered in the molecular diagnostic workup of stroke after exclusion of the JAK2 V617F, particularly in the presence of a thrombocytosis, as a confirmatory finding will impact on future management for the prevention of recurring stroke and other thrombotic episodes.

\section{Conflict of Interest}

None declared.

\section{References}

1 Arachchillage DRJ, Laffan M. Pathogenesis and management of thrombotic disease in myeloproliferative neoplasms. Semin Thromb Hemost 2019;45(06):604-611

2 Stefanou MI, Richter H, Härtig F, et al. Recurrent ischaemic cerebrovascular events as presenting manifestations of myeloproliferative neoplasms. Eur J Neurol 2019;26(06):903-e64

3 Rotunno G, Mannarelli C, Guglielmelli P, et al; Associazione Italiana per la Ricerca sul Cancro Gruppo Italiano Malattie Mieloproliferative Investigators. Impact of calreticulin mutations on clinical and hematological phenotype and outcome in essential thrombocythemia. Blood 2014;123(10):1552-1555

4 Pietra D, Rumi E, Ferretti VV, et al. Differential clinical effects of different mutation subtypes in CALR-mutant myeloproliferative neoplasms. Leukemia 2016;30(02):431-438

5 Pérez Encinas MM, Sobas M, Gómez-Casares MT, et al. The risk of thrombosis in essential thrombocythemia is associated with the type of CALR mutation: a multicentre collaborative study. Eur J Haematol 2021;106(03):371-379

6 Verger E, Crassard I, Cassinat B, Bellucci S. Low incidence of CALR gene mutations in patients with cerebral venous thrombosis without overt chronic myeloproliferative neoplasm. Thromb Res 2015;136(04):839-840

7 Chen CC, Hsu CC, Huang CE, et al. Enhanced risk for specific somatic myeloproliferative neoplastic mutations in patients with stroke. Curr Neurovasc Res 2017;14(03):222-231

8 Curto-Garcia N, Ianotto JC, Harrison CN. What is pre-fibrotic myelofibrosis and how should it be managed in 2018? $\mathrm{Br} \mathrm{J}$ Haematol 2018;183(01):23-34

9 Li M, De Stefano V, Song T, et al. Prevalence of CALR mutations in splanchnic vein thrombosis: a systematic review and metaanalysis. Thromb Res 2018;167:96-103 